International Journal of Engineering \& Technology, 7 (2.12) (2018) 5-7
SPC
Sebsite w ww. sciencepubco.com/index.php/IJET
Research Paper

\title{
Anti-glare coating glass with sol-gel process
}

\author{
Ki-Chul Kim * \\ Department of Advanced Chemical Engineering, Mokwon University, 88 Doanbook-ro, Seo-gu, Daejeon, 35349, Republic of KOREA \\ *Corresponding author E-mail: Kckim30@mokwon.ac.kr
}

\begin{abstract}
Background/Objectives: The next generation car such as self-driving car, and electrical vehicle is a hot issue of the automobile industry, which has the infotainment system with the multi-touch screen panel.

Methods/Statistical analysis: The reflection of light from the surface of touch-panel display hinders the clean image acquisition of the display of infotainment system. In this research, anti-glare coating was fabricated by slot die method with the synthesized sols using the TEOS and functional binder on cleaned slide glass. The morphology and surface roughnesswere characterized by FE-SEM, and AFM. The optical properties of anti-glare coating were analyzed by UV-visible spectrophotometer and haze meter.

Findings: The average transmittance of anti-glare coating glasses exhibits $88.5 \%-90.5 \%$ in the visible light range of $400 \mathrm{~nm}$ to $800 \mathrm{~nm}$ wavelength. The haze value of the anti-glare coating has $7.1 \%-36.6 \%$ to various coating conditions. The haze values of anti-glare coating were mainly affected by coated sol conditions rather than the coating conditions. The coating conditions of slot die method such as gap distance, and discharge rate of nozzle not affect the quality of anti-glare coating. The haze of anti-glare coating was affected by particle density, and particle size. The anti-glare coating of dense particle distribution and large particle exhibit large haze value about $37 \%$.The best performance of anti-glare coatingwas $7.1 \%$ haze, $90.5 \%$ average transmittance, and $11 \mathrm{~nm}$ average surface roughness, which was coated with the solution \#2 sol. The reflectance of anti-glare coating was not affected by change of haze values. But the transmittance of anti-glare coating was affected by haze.

Improvements/Applications: The anti-glare coating glass can be applied to various applications such as protective glass of crystalline $\mathrm{Si}$ photovoltaic cells, window of skyscraper, and roof of airport buildings.
\end{abstract}

Keywords: Anti-Glare Coating; Sol-Gel Process; Slot Die Coating; Touch Panel; Infotainment System.

\section{Introduction}

The next generation car such as self-driving car, connected car, smart car, and electrical vehicle is a hot issue of the automobile industry, which has the infotainment (information + entertainment) system with the multi-touch screen panel. The reflection of light from the surface of touch panel hinders the clean image acquisition of the display of infotainment system as shown in Figure 1. The reduce of reflected light is possible using a various techniques including anti-glare (AG) coating, and anti-reflective (AR) coating. The perfect AR effect can be accomplished by moth-eye structures [1]. The natural AR structure is found in moth-eye which has periodic nanostructures. The biomimetic AR structures were fabricated using a various techniques including colloidal lithography [2], Ag deposition and reactive ion etching (RIE) [3], chemical anisotropic etching ${ }^{4}$, multistep anodization and etching process $^{5}$, hot-embossing method ${ }^{6}$. But the fabrication of the motheye AR structure has drawbacks such as patterning area limit, complex fabrication process, expensive equipment, materials limit etc. The AR coating is commonly realized using a multi-layer thin film coating with vacuum evaporation and sputtering [7], [8]. Recently, various researches for large area facile AR coating techniques were reported using a sol-gel process [9-15].
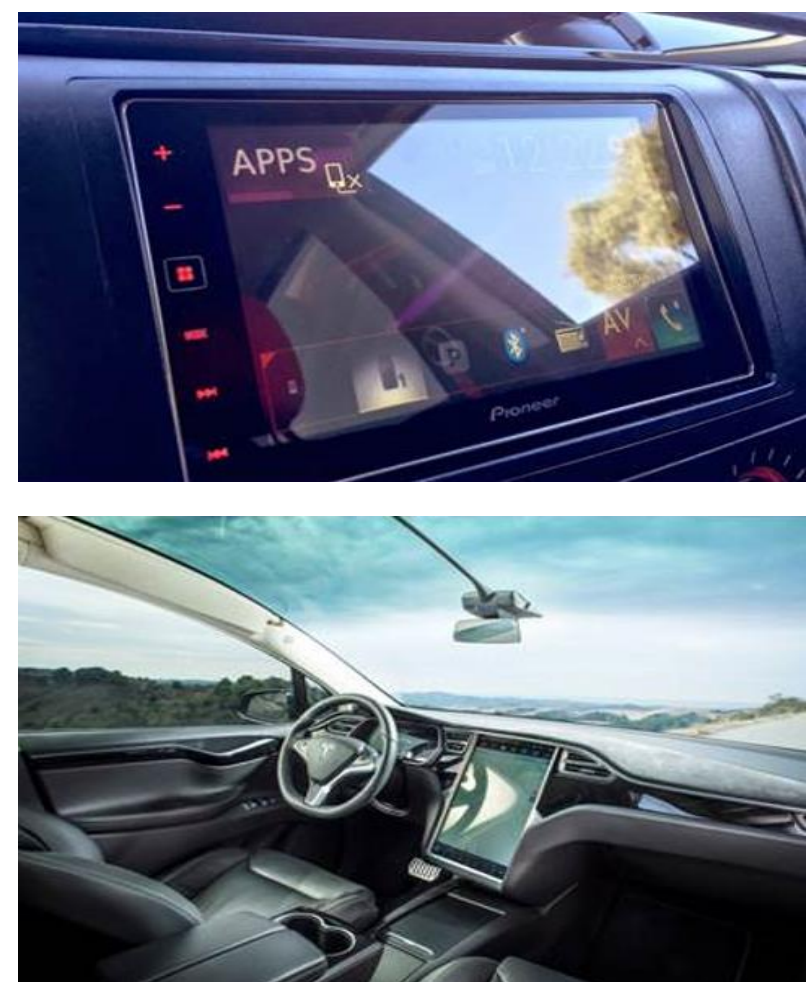

Fig. 1: Digital Photographs Ofcar Infotainment Display. The Reflected Image on Display Surface Hinders Getting the Clear Screen Image. 
The AG coating is a cheaper mass productive technique than the AR coating. The AG coating has the uneven surface which scatter incident light in random direction ${ }^{16}$ as shown in Figure 2. The etching technology based Anti-Glare (AG) coating, using the HF solution, exhibits a good performance but induces the environmental problems. In this research, the AG coating with high scratch-resistance function was fabricated on slide glass substrates by slot die method using a sol solutions which were synthesized sol-gel process. Optical properties such as haze, reflectance, and transmittance were analyzed by UV-visible spectrophotometer, and haze meter. The morphology and surface roughness of the AG coating were characterized by FE-SEM, and AFM.

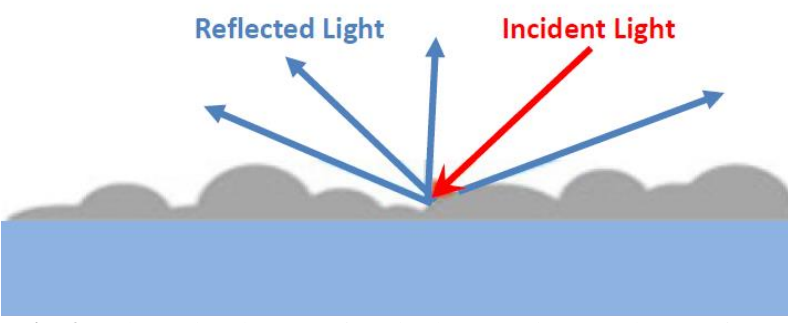

Fig. 2: Schematic Diagram of Anti-Glare Coating on Glass Surface.

\section{Experimental details}

The AG coating films are fabricated commonly with the UV curable binder resin and various fillers including PMMA (polymethyl methacrylate), PS (polystyrene), PE (polyethylene), PP (polypropylene), PU (polyurethane), metal oxide, inorganic and silica nanoparticles on polymer substrate by roll-to-roll process using a micro-gravure, slot die coating method. But the introduce of filler is induced the reducing of hardness of AG coating film. The AG coating of infotainment system require high scratch-resistance. Here, filler free and high scratch-resistance AG coating is reported using a sol-gel process and slot die coating method on slide glass substrate.

The AG solsolutions were synthesized with the mixture of functional haze binder and base binder. The functional haze binder was synthesized with the mixture of 3-(Trimethoxysilyl) propyl Methacrylate (Sigma Aldrich, 98\%), Trimethoxymethylsilane(Sigma Aldrich, 98\%), and Tetraethyl Orthosilicate(Sigma Aldrich, 98\%). The mixing ratio of functional haze binder was the weight percent of $90 \% \sim 50 \%, 50 \% \sim 20 \%$, and $20 \% \sim 10 \%$, respectively. The ammonia solution was dropped to the mixture of haze binder for the adjusting $\mathrm{pH}$. The sol-gel process was proceeded to adding the distilled water. The mixture of functional haze binder was stirred at $50 \sim 60{ }^{\circ} \mathrm{C}$ for $3 \sim 5$ hours, and aging for 24 hours. The base binder was synthesized with the TEOS (Tetraethyl Orthosilicate, Sigma Aldrich, 98\%), and (3-Glycidyloxypropyl)trimethoxysilane (Sigma Aldrich, 98\%). The mixing ratio was the weight percent of $90 \% \sim 70 \%$, and $30 \% \sim 10 \%$. The nitric acid solution was dropped to the mixture of base binder for the adjusting $\mathrm{pH}$. The sol-gel process was proceeded to adding the distilled water. The mixture of base binder was stirred at $30 \sim 40{ }^{\circ} \mathrm{C}$ for 3 hours. The final sol solutions were mixed the haze binder and base binder of 90:10 weight ratio for 2 hours at room temperature.

The final coating sol solutions were diluted with the isopropyl alcohol, and ethanol to final sol solutions for adjusting $5.5 \%$ solid content. The AG coating was coated by slot die method on cleaned slide glass substrate. The coating conditions were gap distance of $50 \mu \mathrm{m}, 300 \mu \mathrm{m}$, and $500 \mu \mathrm{m}$, and discharge rate of nozzle of 5 $\mathrm{ml} / \mathrm{min}, 7 \mathrm{ml} / \mathrm{min}, 11 \mathrm{ml} / \mathrm{min}$, respectively. The optical properties such as haze, and transmittance were characterized by haze meter (Nippon Denshoku, NDH), and UV-visible spectrophotometer (Scinco, Mega-800).

\section{Results and discussion}

The surface morphology of the AG coating was investigated by FE-SEM as shown in Figure 3. The coating conditions of slot die method such as gap distance, and discharge rate of nozzle not affect the quality of AG coating. It means that the surface morphology of AG coating with $50 \mu \mathrm{m}$ gap distance and $5 \mathrm{ml} / \mathrm{min}$ discharge rate of nozzle is equal to one of AG coating with 500 $\mu \mathrm{m}$ gap distance and $7 \mathrm{ml} / \mathrm{min}$ discharge rate of nozzle or $300 \mu \mathrm{m}$ gap distance and $11 \mathrm{ml} / \mathrm{min}$ discharge rate of nozzle. The haze values of AG coating were mainly affected by coated sol conditions rather than the coating conditions. The haze of AG coating was affected by particle density and particle size. The haze of sol solution \#3 was $36.6 \%$ correspond to Figure 3 (c) which exhibited medium particle size, and dense particle distribution. The haze of sol solution \#2 was $7.1 \%$ correspond to Figure 3 (b) which exhibited fine particle size and uniform particle distribution. The haze of sol solution \#1 was $14.0 \%$ correspond to Figure 3 (a) which exhibited large particle size, and sparse particle distribution.
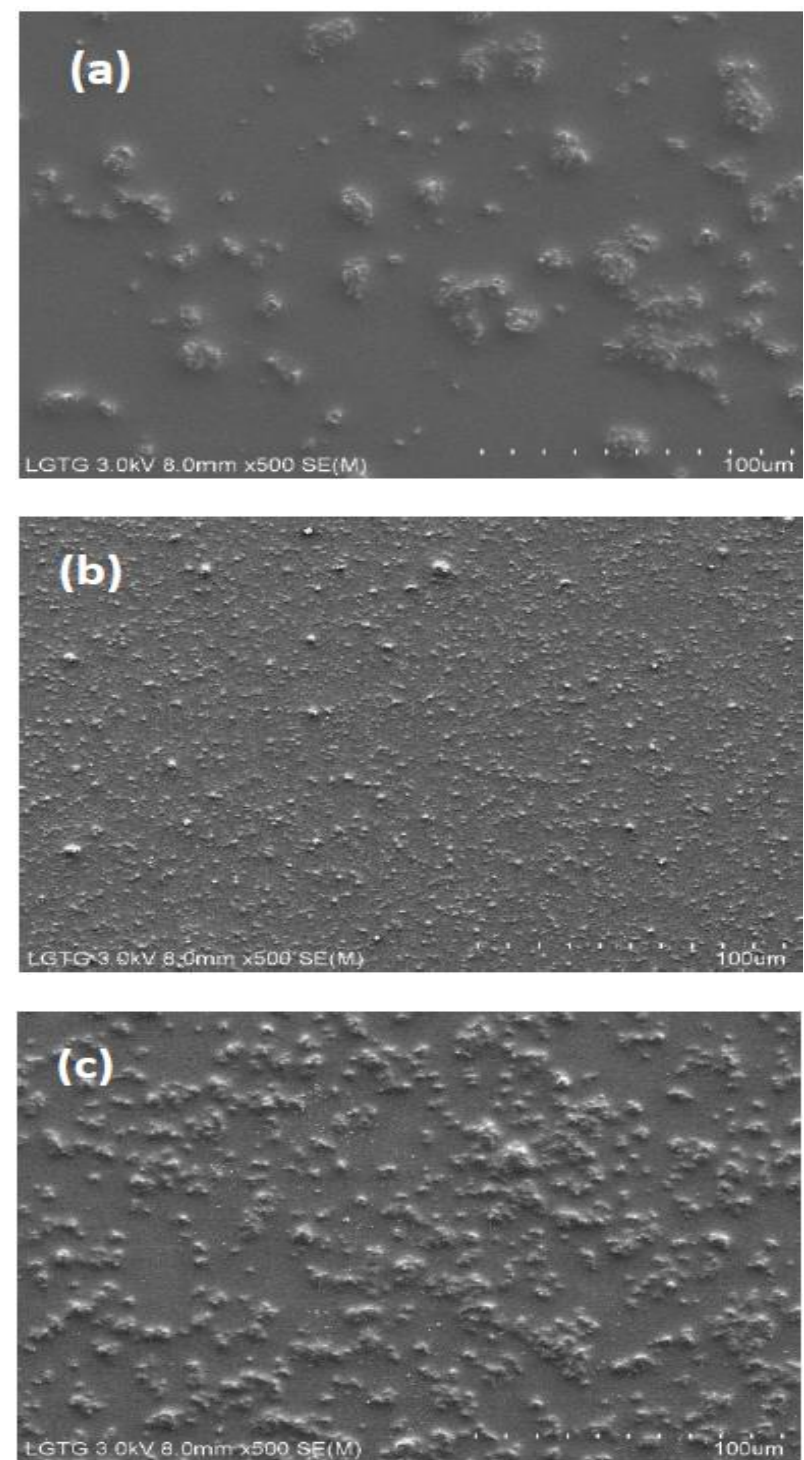

Fig. 3: FE-SEM Images of the Anti-Glare Coating Glass by Slot Die Method with the Condition of $50 \mu \mathrm{M}$ Gap Distance, and $5 \mathrm{Ml} / \mathrm{Min}$ Discharge Rate of Nozzle Using A Synthesized Sol (A) Solution \#1, (B) Solution \#2, and (C) Solution \#3 on Cleaned Slide Glass Substrate. The Magnification Is 500 .

The AG coating is required to moderate haze and high transmittance. The best performance of AG coating was $7.1 \%$ haze, $90.5 \%$ average transmittance and $11 \mathrm{~nm}$ average surface roughness as shown in Figure $4 \&$ 5, which was coated with the solution \#2 sol 
which exhibited fine particle size and uniform particle distribution. The reflectance of AG coating was not affected by change of haze values. The average reflectance of AG coating of sol solution $\# 1$, \#2, and \#3 is equal to $7.9 \%$. But the transmittance of AG coating was affected by the haze values. The average transmittance of sol solution \#1, \#2, and \#3 was $89.8 \%, 90.5 \%$, and $88.5 \%$, respectively.

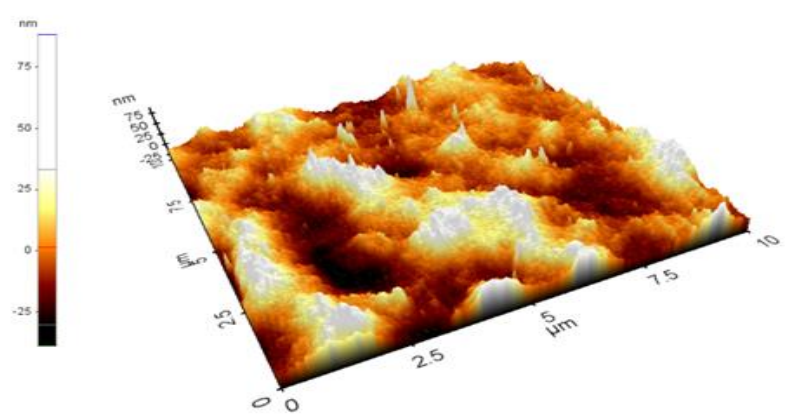

Fig. 4: AFM Image of the Anti-Glare Coating Glass by Slot Die Method with the Condition of $50 \mu \mathrm{M}$ Gap Distance, And 5 Ml/Min Discharge Rate of Nozzle Using a Synthesized Sol Solution \#2.

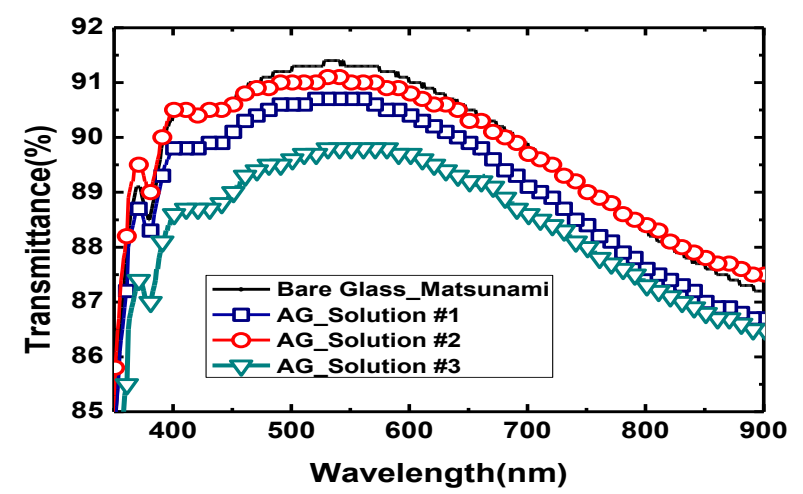

Fig. 5: Transmittance Curves of the Bare Slide Glass and Anti-Glare Coating Glasses by Slot Die Method.

The digital photograph of comparing reflected image betweenAG coating glass and non-AG coating glass was shown in Figure 6. The reflected image on AG coating is more diffused which means anti-glare effect.

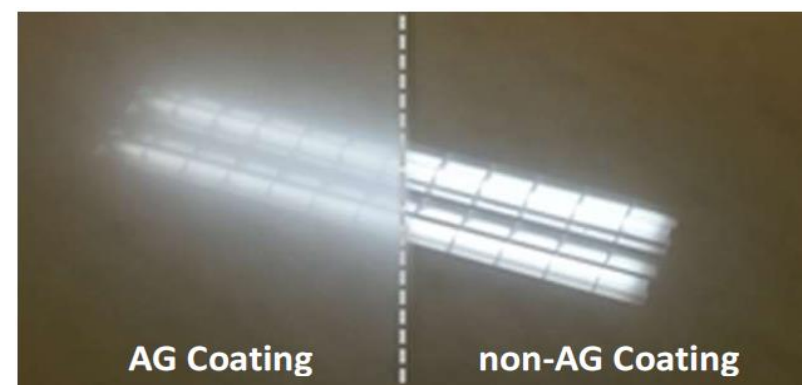

Fig. 6: Digital Photograph of Comparing Reflected Image between AntiGlare Coating Glass and Non-AG Coating Bare Glass.

\section{Conclusion}

The Anti-Glare coating glass was fabricated by slot die method using a synthesized sol solutions. The haze values are mainly affected by particle distribution and particle size. The large haze value is attributed to dense particle distribution, and medium or large particle size. The AG coating glass can be applied to various applications such as protective glass of crystalline silicon photovoltaic cells, window of skyscraper, and roof of airport buildings. The AG coating glass is a one of solutions for light pollution of skyscraper, and hindering of airplane controlby reflected light from the roof of airport buildings.

\section{Acknowledgment}

This work (Grant No. S2470403) was supported by the Starting growth Technological R\&D Program \&Korea Technology and Information Promotion Agency for SMEs (TIPA) funded by Ministry of SMEs and Startups of the Korea government.

\section{References}

[1] Stavenga D G, Foletti S, Palasantzas G, Arikawa K, Light on the moth-eye corneal nipple array of butterflies, Proceedings of the Royal Society B, 2006, 273, pp. 661-667.

[2] Zhang X, Zhang J, Ren Z, Li X, Zhang X, Zhu D, Wang T, Tian $\mathrm{T}$, Yang B, Morphology and wettability control of silicon cone array using colloidal lithography, Langmuir, 2009, 25(13), pp. 7375-7382.

[3] Wang S, Yu X Z, Fan H T, Simple lithographic approach for subwavelength structure antireflection, Applied Physics Letters, 2007, 91, p. 061105 .

[4] Papet P, Nichiporuk O, Kaminski A, Rozier Y, Kraiem J, Lelievre J F, Chaumartin A, Fave A, Lemiti M, Pyramidal texturing of silicon solar cell with TMAH chemical anisotropic etching, Solar Energy Materials \& Solar Cells, 2006, 90, pp. 2319-2328.

[5] Choi K, Park S H, Song Y M, Lee Y T, Hwangbo C K, Yang H, Lee H S, Nano-tailoring the surface structure for the monolithic high-performance antireflection polymer film, Advanced Materials, 2010, 22, pp. 3713-3718

[6] Han K S, Lee H, Kim D, Lee H, Fabrication of anti-reflection structure on protective layer of solar cells by hot-embossing method, Solar Energy Materials \& Solar Cells, 2009, 93, pp. 1214-1217.

[7] Kuo M L, Poxson D J, Kim Y S, Mont F W, Kim J K, Schubert E F, Lin S Y, Realization of a near-perfect antireflection coating for silicon solar energy utilization, Optics Letters, 2008, 33(21), pp. 2527-2529.

[8] Kim K C, Effective graded refractive-index anti-reflection coating for high refractive-index polymer ophthalmic lenses, Materials Letters, 2015, 160, pp. 158-161.

[9] Moghal J, Kobler J, Sauer J, Best J, Gardener M, Watt A A R, Wakefield G, High-performance, single-layer antireflective optical coatings comprising mesoporous silica nanoparticles, ACS Applied Materials \& Interfaces, 2012, 4, pp. 854-859.

[10] Glaubitt W, Löbmann P, Anti-soiling effect of porous $\mathrm{SiO} 2$ coatings prepared by sol-gel processing, Journal of Sol-Gel Science Technology, 2011, 59, pp. 239-244.

[11] Liu B T, Yeh W D, Antireflective surface fabricated from colloidal silica nanoparticles, Colloids and Surfaces A: Physicochemical Engineering Aspects, 2010, 356, pp. 145-149.

[12] Kim K C, Dependence of surface morphology of transparent hydrophobic anti-reflective coating, Journal of the Korea Academia-Industrial cooperation Society, 2017, 18(10), pp. 771-776.

[13] Lee D, Fubner M F, Cohen R E, All-nanoparticle thin-film coating, Nano Letters, 2006, 6(10), pp. 2305-2312.

[14] Moghal J, Reid S, Hagerty L, Gardener M, Wakefield G, Development of single layer nanoparticle anti-reflection coating for polymer substrates, Thin Solid Films, 2013, 534, pp. 541-545.

[15] Askar K, Phillips B M, Fang Y, Choi B, Gozubenli N, Jiang P, Jiang B, Self-assembled self-cleaning broadband anti-reflection coatings, Colloids and Surfaces A: Physicochemical Engineering Aspects, 2013, 439, pp. 84-100.

[16] Nuijs A M, Horikx J J L, Diffraction and scattering at antiglare structures for display devices, Applied Optics, 1994, 33(18), pp. 4058-4068. 